NOTA CIENTÍFICA

\title{
Toxicidad aguda y crónica del lindano sobre Ceriodaphnia cornuta (Cladocera: Daphniidae)
}

\author{
Acute and chronic toxicity of lindane on Ceriodaphnia cornuta (Cladocera: \\ Daphniidae)
}

\author{
Jorgelina Juárez y Alcira Villagra de Gamundi
}

\begin{abstract}
Instituto de Limnología (ILINOA), Facultad de Ciencias Naturales e Instituto Miguel Lillo, Universidad Nacional de Tucuman, Argentina. E-mail Jorgelina Juárez: jorgelina」@yahoo.com
\end{abstract}

$\begin{array}{ll}\text { Presentado: } & 18 / 08 / 2009 \\ \text { Aceptado: } & 23 / 07 / 2010 \\ \text { Publicado online: } & 21 / 01 / 2011\end{array}$

\section{Resumen}

El lindano es un plaguicida organoclorado cuya toxicidad produce efectos nocivos en la salud humana y la biota. El objetivo de este estudio fue evaluar la toxicidad aguda y crónica del lindano sobre el microcrustáceo Ceriodaphnia cornuta. Para las pruebas agudas se empleó un diseño estático, usando 10 neonatos $\leq 24$ horas de edad para el control y para cada concentración de lindano (5, 10, 15, 20 y $25 \mathrm{mg} / \mathrm{L})$. Se realizaron tres réplicas de cada tratamiento. Se controló la inmovilización de los individuos a las 48 hs y se calcularon los valores de $\mathrm{CL}_{50}$. Los bioensayos crónicos consistieron en un diseño semi-estático, utilizando 10 neonatos menores de 24 horas de edad (uno por recipiente) para el control y para cada concentración subletal del tóxico $(0,1 ; 0,15 ; 0,2 ; 0,25$ y $0,3 \mathrm{mg} / \mathrm{L})$. Se evaluaron los efectos sobre la supervivencia y reproducción durante 21 días. El valor obtenido de $\mathrm{CL}_{50} 48$ horas fue de 5,293 $\pm 0,7 \mathrm{mg} / \mathrm{L}$. Los parámetros reproductivos (neonatos por hembra, tamaño de la camada e índice de incremento natural) disminuyeron al aumentar la concentración del tóxico evaluado, mientras que el inicio de la madurez sexual se retrasó, demostrando la sensibilidad de los organismos prueba al lindano.

Palabras claves: Toxicidad aguda, Toxicidad crónica, Lindano, Ceriodaphnia cornuta

\section{Abstract}

Lindane is an organochlorine pesticide toxicity which produces harmful effects on human health and biota. The aim of this study was to evaluate the acute and chronic toxicity of Lindane on microcrustaceans Ceriodaphnia cornuta. For acute tests used a static design, using 10 infants $\leq 24$ hours of age for control and each concentration of lindane $(5,10,15,20$ and $25 \mathrm{mg} / \mathrm{L})$. There were three replicates of each treatment. Monitored the detention of individuals at 48 am and calculated LC50 values. Chronic Bioassays consisted of a semi-static design, using 10 infants $\leq 24$ hours of age (one per vessel) for the control and each of the toxic sublethal concentration $(0.1,0.15,0.2,0.25$ and $0.3 \mathrm{mg} / \mathrm{L})$. The effects on survival and reproduction over 21 days. The $\mathrm{LC}_{50}$ value of 48 hours was $5.293 \pm 0.7 \mathrm{mg} / \mathrm{L}$. Reproductive parameters (neonates per female, litter size and rate of natural increase) decreased with increasing concentration of toxic evaluated, while the onset of sexual maturity is delayed, thus demonstrating the sensitivity of test organisms to lindane.

Keywords: Acute Toxicity, Chronic Toxicity, Lindane, Ceriodaphnia cornuta.

\section{Introducción}

El lindano ( $\boldsymbol{Y}$-hexaclorociclohexano) es un plaguicida organoclorado que produce alteraciones orgánicas, genéticas y reproductivas en la biota; cabe señalar también, que su afinidad con los lípidos permite su bioacumulación y bioconcentración a través de las cadenas tróficas (Ávalos Gómez et al. 2003). Es usado principalmente para el control de plagas en vegetales encontrándose entre los agroquímicos de más frecuente uso. Según la Agencia de Protección Ambiental (EPA) se incluye entre los compuestos de toxicidad moderada clase II. Esta sustancia está prohibida en numerosos países como Australia, Austria, Indonesia, Nueva Zelanda, Holanda, Canadá y EE.UU. (INE 2004), por el riesgo ecológico y efectos adversos que causa en la fauna terrestre y acuática (Iannacone et al. 2000). En Argentina el lindano está restringido a distintos usos: a) En Sanidad Animal (Decreto 2143/68) y más tarde se prohibió completamente su uso veterinario (Resolución 513/98), b) En Sanidad Vegetal (Disp. 80/71), después se lo prohibió como gorgojicida (Disp. 47/72) como también completamente su uso en vegetales (Resolución 513/98) y c) Como Insecticida Domisanitario (Resolución 7292/98) (Giorgio et al. 2005). Por esta razón es importante determinar el nivel de riesgo ambiental del plaguicida lindano sobre organismos representantes del ecosistema acuático utilizando bioensayos de toxicidad (Iannacone et al. 2000).
Los cladóceros son ampliamente empleados en bioensayos de toxicidad, debido a importantes características como: reproducción partenogenética, corto tiempo generacional, alta resistencia a cambios ambientales y como organismo filtrador poco selectivo (Villarroel Utrillas 2004), que permiten disponer de abundantes especímenes idénticos en un tiempo relativamente reducido. En tal sentido los individuos cultivados presentan las condiciones fisiológicas óptimas para el desarrollo de la prueba de toxicidad, además Ceriodaphnia cornuta es una de las tres especies de cladóceros más abundantes en los cuerpos de aguas tropicales, adaptado a tolerar bajas concentraciones de oxígeno y altas variaciones de temperatura, distribuyéndose ampliamente en diversos humedales (Villalobos et al. 2006), lo que favorece su uso como organismos prueba.

El objetivo del presente estudio fue evaluar la toxicidad aguda y crónica del lindano sobre neonatos $(<24$ horas de edad) del cladócero Ceriodaphnia cornuta.

\section{Condiciones de laboratorio}

Los organismos prueba (Ceriodaphnia cornuta G.O. Sars, 1885) fueron colectados del Embalse Celestino Gelsi - Tucumán y se cultivaron en el laboratorio del Instituto de Limnología del Noroeste Argentino perteneciente a la Facultad de Ciencias Naturales e Instituto Miguel Lillo (Universidad Nacional de 
Tabla 1. Condiciones de prueba de toxicidad aguda.

\begin{tabular}{ll}
\hline Factor & Condición \\
\hline Temperatura & $25 \pm 2{ }^{\circ} \mathrm{C}$ \\
Fotoperíodo & $12: 12 \mathrm{~h} \mathrm{Luz} /$ oscuridad \\
Envase prueba & $25 \mathrm{~mL}$ \\
Volumen de exposición & $15 \mathrm{~mL}$ \\
Edad de organismos prueba & Neonatos ? 24 h \\
$\mathrm{N}^{o}$ de réplicas por concentración & 3 \\
$\mathrm{~N}^{o}$ de concentraciones más control & $6(5,10,15,20$ y $25 \mu \mathrm{g} / \mathrm{mL})$ \\
Organismos por envase & 10 \\
Organismos totales por concentración & 30 \\
Alimentación & No requiere \\
Agua de dilución & Agua natural filtrada \\
Tiempo de exposición & $48 \mathrm{~h}$ \\
Respuesta letal & Mortalidad, sin movilidad \\
\hline
\end{tabular}

Tucumán - Tucumán - Argentina). Se mantuvieron en laboratorio en acuarios de 10 litros de capacidad, bajo condiciones de laboratorio de temperatura ambiente $\left(25 \pm 2{ }^{\circ} \mathrm{C}\right)$, con un fotoperiodo natural de aproximadamente 12 horas luz/oscuridad y un $\mathrm{pH}$ constante de 7 . La frecuencia de alimentación fue de 2 veces por semana con jugo de salvado de avena $(1 \mathrm{~mL}$ de concentrado/L de medio) y la de limpieza de mudas y desechos, semanalmente. Las concentraciones del tóxico fueron preparadas a partir de una solución stock proporcionada por el Instituto de Evaluación y Control de contaminantes Ambientales (LECCA) de la Facultad de Ciencias Exactas y Tecnología de la Universidad Nacional de Tucumán.

\section{Diseño de experimento}

Las pruebas agudas fueron realizadas de acuerdo con el protocolo estándar (ISO 1982), iniciándose con neonatos, menores de 24 horas de edad, obtenidos de cultivos en masa y alimentados con jugo de salvado de avena. Se empleó un diseño estático, usando 10 individuos para el control y para cada concentración de lindano $(5,10,15,20$ y $25 \mathrm{mg} / \mathrm{L})$. Los recipientes de prueba consistieron (tres réplicas por cada tratamiento) en tubos de

Tabla 2. Condiciones de prueba de toxicidad crónica.

\begin{tabular}{ll}
\hline Factor & Condición \\
\hline Temperatura & $25 \pm 2{ }^{\circ} \mathrm{C}$ \\
Fotoperíodo & $12: 12 \mathrm{~h} \mathrm{Luz} /$ oscuridad \\
Envase prueba & $25 \mathrm{~mL}$ \\
Volumen de exposición & $15 \mathrm{~mL}$ \\
Edad de organismos prueba & Neonatos $\leq 24 \mathrm{~h}$ \\
$\mathrm{~N}^{o}$ de réplicas por concentración & 10 \\
$\mathrm{~N}^{o}$ de concentraciones más control & $6(0,1 ; 0,15 ; 0,2 ; 0,25$ y 0,3 \\
Organismos por envase & $1 \mathrm{~g} / \mathrm{mL})$ \\
Organismos totales por concentración & 10 \\
Renovación de solución test & semanalmente \\
Alimentación & 2 veces por semana \\
Agua de dilución & Agua natural filtrada \\
Tiempo de exposición & 21 días \\
& Neonatos por hembra, \\
& tamaño de la camada, \\
Parámetros & madurez sexual, \\
& longevidad e índice de \\
& incremento natural. \\
\hline
\end{tabular}

ensayos de $25 \mathrm{~mL}$ que contienen $15 \mathrm{~mL}$ de agua de dilución (medio de cultivo). (Tabla 1). Se controló la inmovilización de los individuos en todos los tratamientos experimentales a las $48 \mathrm{~h}$ y se contabilizaron para la determinación posterior de los valores de $\mathrm{CL}_{50}$ (concentración que produce el 50\% de mortalidad), mediante el programa estadístico Probit (US EPA 1988).

Para los ensayos crónicos se empleó un diseño semi-estático, utilizando 10 neonatos de aproximadamente 24 hs de edad (uno por recipiente) para el control y para cada concentración subletal de lindano $(0,1 ; 0,15 ; 0,2 ; 0,25$ y $0,3 \mathrm{mg} / \mathrm{L})$. Los recipientes experimentales fueron iguales a los utilizados en los ensayos agudos. Se evaluaron los efectos sobre la supervivencia y reproducción durante 21 días (neonatos por hembra, tamaño de la camada, edad de madurez sexual, longevidad e índice de incremento natural). (Tabla 2). Los neonatos originados fueron contados y desechados.

Durante el período de monitoreo se mantuvieron las condiciones de laboratorio y los patrones rutinarios de alimentación y limpieza establecidos para los cultivos masivos.

Los parámetros demográficos se calcularon usando las siguientes fórmulas:

Tasa reproductiva neta $\left(\mathrm{R}_{0}\right)=\sum \mathrm{l}_{\mathrm{x}} \mathrm{m}_{\mathrm{x}}$

Tiempo generacional $(\mathrm{Tg})=\sum \mathrm{l}_{\mathrm{x}} \mathrm{m}_{\mathrm{x}} \mathrm{x} / \mathrm{R}_{0}$

Tasa intrínseca de crecimiento natural $(r)=\ln \mathrm{R}_{0} / \mathrm{T}$

Donde:

$\mathrm{m}_{\mathrm{x}}=\mathrm{Al}$ número de neonatos producidos por hembras sobrevivientes a la edad $\mathrm{x}$

$l_{\mathrm{x}}=$ La proporción de individuos sobrevivientes a la edad $\mathrm{x}$ $\mathrm{x}=$ Días

Los datos se analizaron estadísticamente usando el análisis de varianza (ANOVA) para determinar las diferencias significativas entre el control y los distintos tratamientos.

\section{Resultados}

En la Tabla 3 y Figura 1 se muestran los porcentajes de mortalidad observados de neonatos menores de $24 \mathrm{~h}$ de edad de $C$. cornuta para cada una de las concentraciones de lindano y los valores obtenidos de $\mathrm{CL}_{50}$ a 24 y 48 h de exposición.

La Tabla 4 muestra los efectos del lindano sobre la reproducción y supervivencia de C. cornuta. Se observa un incremento gradual de la edad a la que los organismos alcanzan la madurez sexual, valores promedios comprendidos entre 10,2 días para el control y de 14 días para la máxima concentración (0,3 mg/L) (Fig. 2).

Tabla 3. Porcentaje de mortalidad y $\mathrm{CL}_{50}$ a 24 y 48 h de exposición.

\begin{tabular}{ccc}
\hline \multirow{2}{*}{$\begin{array}{c}\text { Concentraciones } \\
\text { Lindano }(\mathbf{m g} / \mathrm{L})\end{array}$} & \multicolumn{2}{c}{$\%$ Mortalidad } \\
\cline { 2 - 2 } Control & $\mathbf{4 8} \mathbf{h}$ de exposición & $\mathbf{2 4} \mathbf{h}$ de exposición \\
\hline $\mathbf{5}$ & 40 & 57 \\
$\mathbf{1 0}$ & 63 & 63 \\
$\mathbf{1 5}$ & 73 & 73 \\
$\mathbf{2 0}$ & 83 & 93 \\
$\mathbf{2 5}$ & 97 & 100 \\
$\mathrm{CL}_{50}$ & 100 & 100 \\
\hline
\end{tabular}




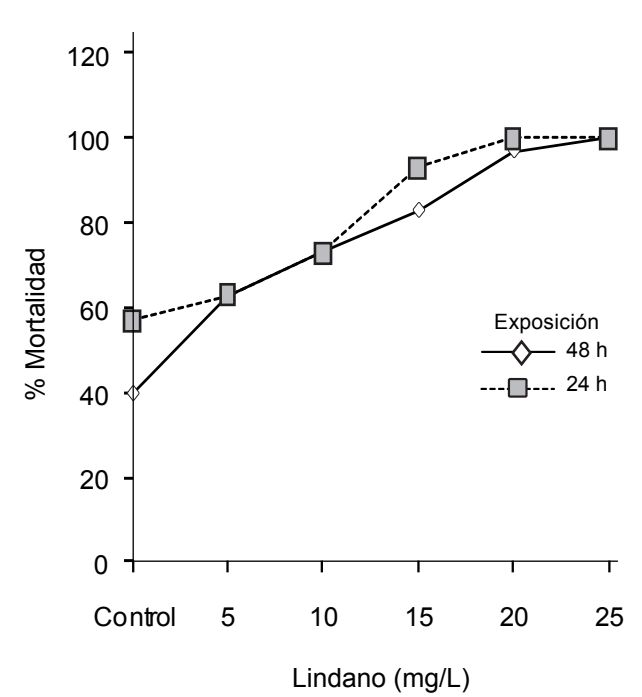

Figura 1. Mortalidad de Ceriodaphnia cornuta a 24 y 48 h de exposición.

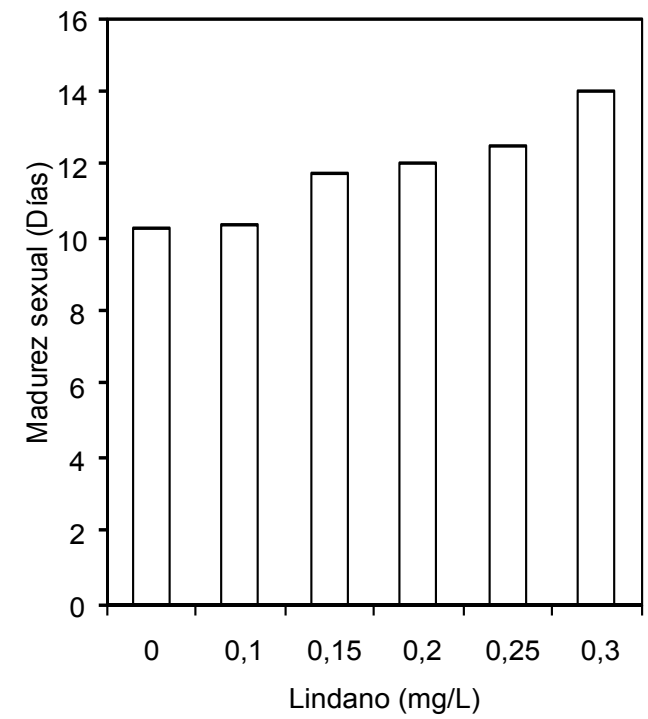

Figura 2. Efectos del lindano sobre la madurez sexual de Ceriodaphnia cornuta.

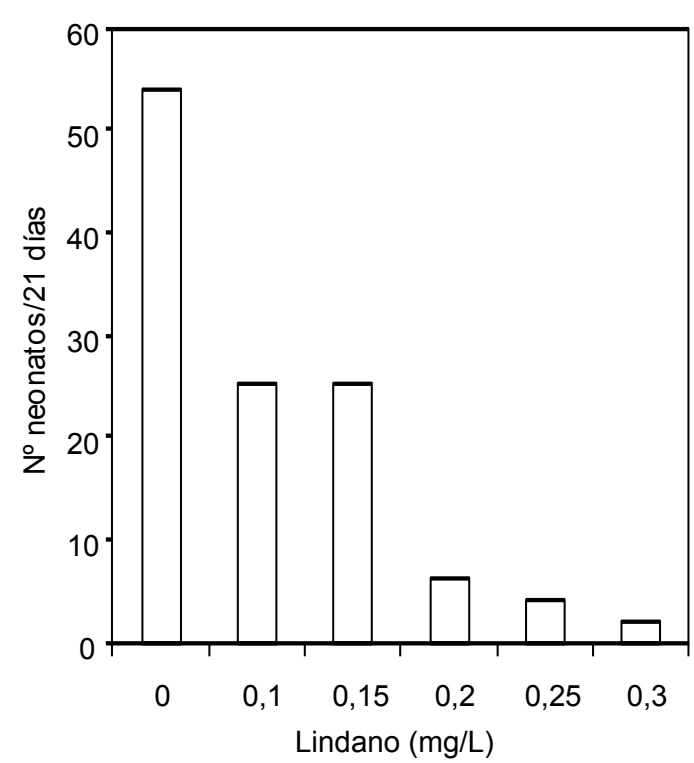

Figura 3. Producción de neonatos de Ceriodaphnia cornuta después de 21 días de tratamiento con lindano.

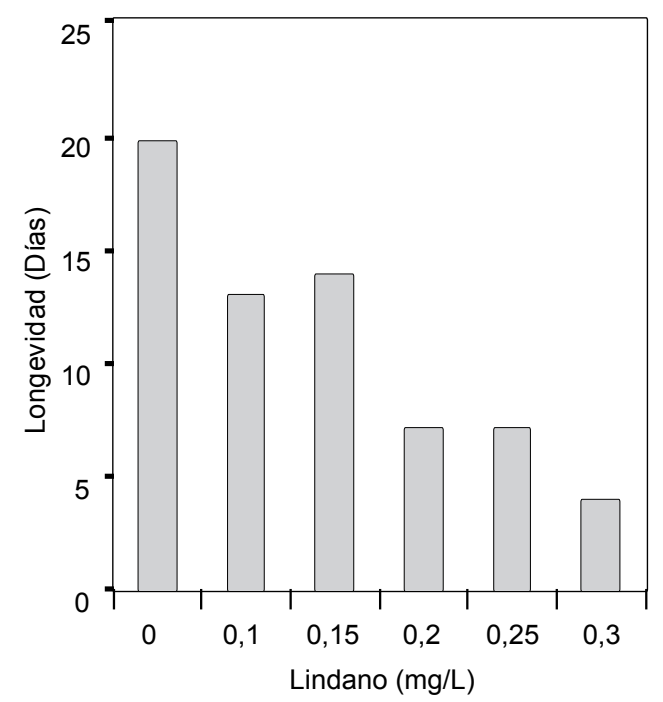

Figura 4. Efectos del lindano sobre la longevidad de Ceriodaphnia cornuta.

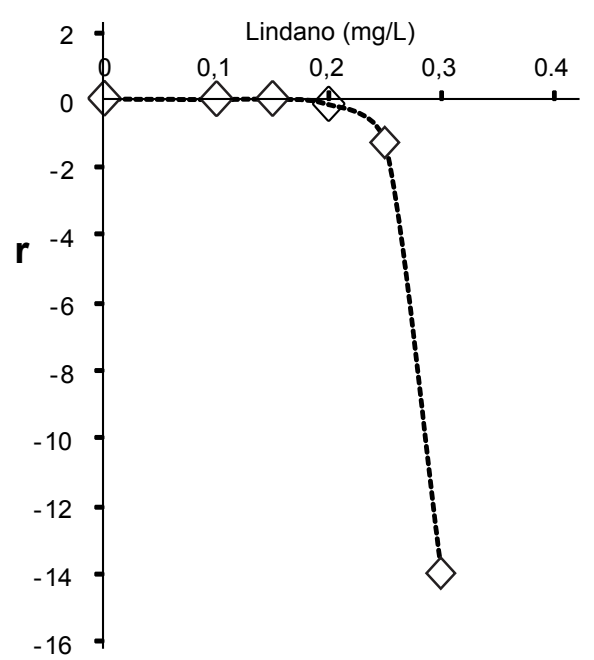

Figura 5. Tasa intrínseca de crecimiento natural (r) de Ceriodaphnia cornuta expuesta a diferentes concentraciones de lindano.

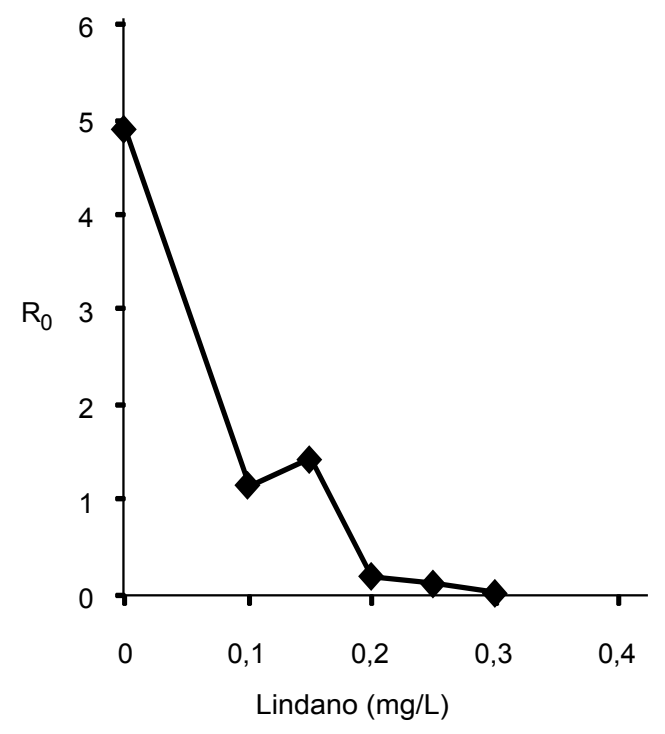

Figura 6. Tasa reproductiva neta $\left(R_{0}\right)$ de Ceriodaphnia cornuta expuesta a diferentes concentraciones de lindano. 
Tabla 4. Efectos del lindano sobre el ciclo de vida y parámetros demográficos de Ceriodaphnia cornuta.

\begin{tabular}{lcccccc}
\hline & \multicolumn{5}{c}{ Lindano (mg/L) } \\
\cline { 2 - 6 } & $\mathbf{0}$ & $\mathbf{0 , 1}$ & $\mathbf{0 , 1 5}$ & $\mathbf{0 , 2}$ & $\mathbf{0 , 2 5}$ & $\mathbf{0 , 3}$ \\
\hline Madurez sexual (Días) & 10,2 & 10,3 & 11,7 & 12 & 12,5 & 14 \\
$\mathbf{N}^{\mathbf{0}}$ neonatos/21 días & 54 & 25 & 25 & 6 & 4 & 2 \\
$\mathbf{N}^{\mathbf{0}}$ neonatos/día & 2,6 & 1,2 & 1,2 & 0,3 & 0,2 & 0,1 \\
Neonatos/adulto $_{\text {Longevidad (Días) }}$ & 5,4 & 2,5 & 2,5 & 0,6 & 0,4 & 0,2 \\
$\mathbf{R}_{\mathbf{0}}$ & 19,8 & 13 & 13,8 & 7,4 & 7 & 4 \\
Tg & 4,91 & 1,16 & 1,43 & 0,2 & 0,12 & 0,02 \\
$\mathbf{r}$ & 13 & 12 & 14 & 11 & 10 & 14 \\
\hline
\end{tabular}

Despues de 21 días de prueba, Ceriodaphnia cornuta produjo un número considerablemente menor de neonatos a mayor concentración del toxico, aunque a concentraciones de 0,1 y 0,15 $\mathrm{mg} / \mathrm{L}$ de lindano dieron el mismo resultado (Fig. 3). Así mismo, la longevidad decrece a medida que aumenta la concentración del plaguicida, a excepción de los grupos experimentales 0,1 y 0,15 $\mu \mathrm{g} / \mathrm{mL}$ con 13 y 13,8 días de supervivencia respectivamente, (Fig. 4). Los resultados para la tasa intrínseca de crecimiento natural (r) se muestran en la Figura 5. Se observa una acentuada disminución a partir de la concentración de $0,2 \mathrm{mg} / \mathrm{L}$. Esta disminución es particularmente considerable a la máxima concentración del lindano $(0,3 \mathrm{mg} / \mathrm{L})$ con un valor de $(\mathrm{r})$ de $-13,97$. La tasa reproductiva neta (Fig. 6) mostró valores decrecientes desde el control $(4,91)$ a la máxima concentración $(0,02)$.

El análisis estadístico de los resultados (ANOVA) puso de manifiesto diferencias significativas entre el control y los tratamientos en la edad de la primera reproducción $(\mathrm{p}=0,0048)$, producción de neonatos en 21 días $(\mathrm{p}=0,0027)$ y longevidad $(\mathrm{p}=$ 0,0012 ) y no mostró diferencias significativas en los parámetros (r) tasa intrínseca de crecimiento natural $(\mathrm{p}=0,1514)$ y $(\mathrm{Tg})$ tiempo generacional $(\mathrm{p}=0,7749)$.

\section{Discusión y conclusiones}

Los resultados obtenidos permiten hacer algunas consideraciones respecto a la eficiencia de $C$. cornuta como organismo prueba en bioensayos de toxicidad.

Los ensayos agudos evidenciaron que $C$. cornuta tiene una menor sensibilidad al lindano, con un valor de $\mathrm{CL}_{50} 48 \mathrm{~h}(5,293$ $\pm 0,7 \mathrm{mg} / \mathrm{L})$ respecto a otros dáfnidos de mayor tamaño como Simocephalus vetulus (11,4 $\mu \mathrm{g} / \mathrm{L}$ ) (Juárez et al. 2007) y Daphnia magna $(460 \mu \mathrm{g} / \mathrm{L})($ Silva et al. 2001) y (Martin et al. 2007). También es más tolerante en comparación con los valores registrados para Daphnia magna y Daphnia longispina (Antunes et al. 2004) para lindano con LC $_{50} 48 \mathrm{~h}$ que oscilaron entre 1802 y 2843 $\mu \mathrm{g} / \mathrm{L}$ y 2079 y $3116 \mu \mathrm{g} / \mathrm{L}$ respectivamente, a distintos niveles de alimentación con algas. Villarroel Utrillas (2004), describió un valor para el organoclorado Propanil sobre $D$. magna de $5,02 \mathrm{mg} / \mathrm{L}$, valor comparativamente similar al obtenido en el presente estudio. El porcentaje de mortalidad a las 24 y $48 \mathrm{~h}$ observó un rango de $40-100 \%$ y $57-100 \%$ respectivamente (concentraciones de 0 a $25 \mathrm{mg} / \mathrm{L}$ ).

La Figura 8 expresa la relaciones integradoras de los parámetros demográficos estudiados pudiéndose observar la sensibilidad

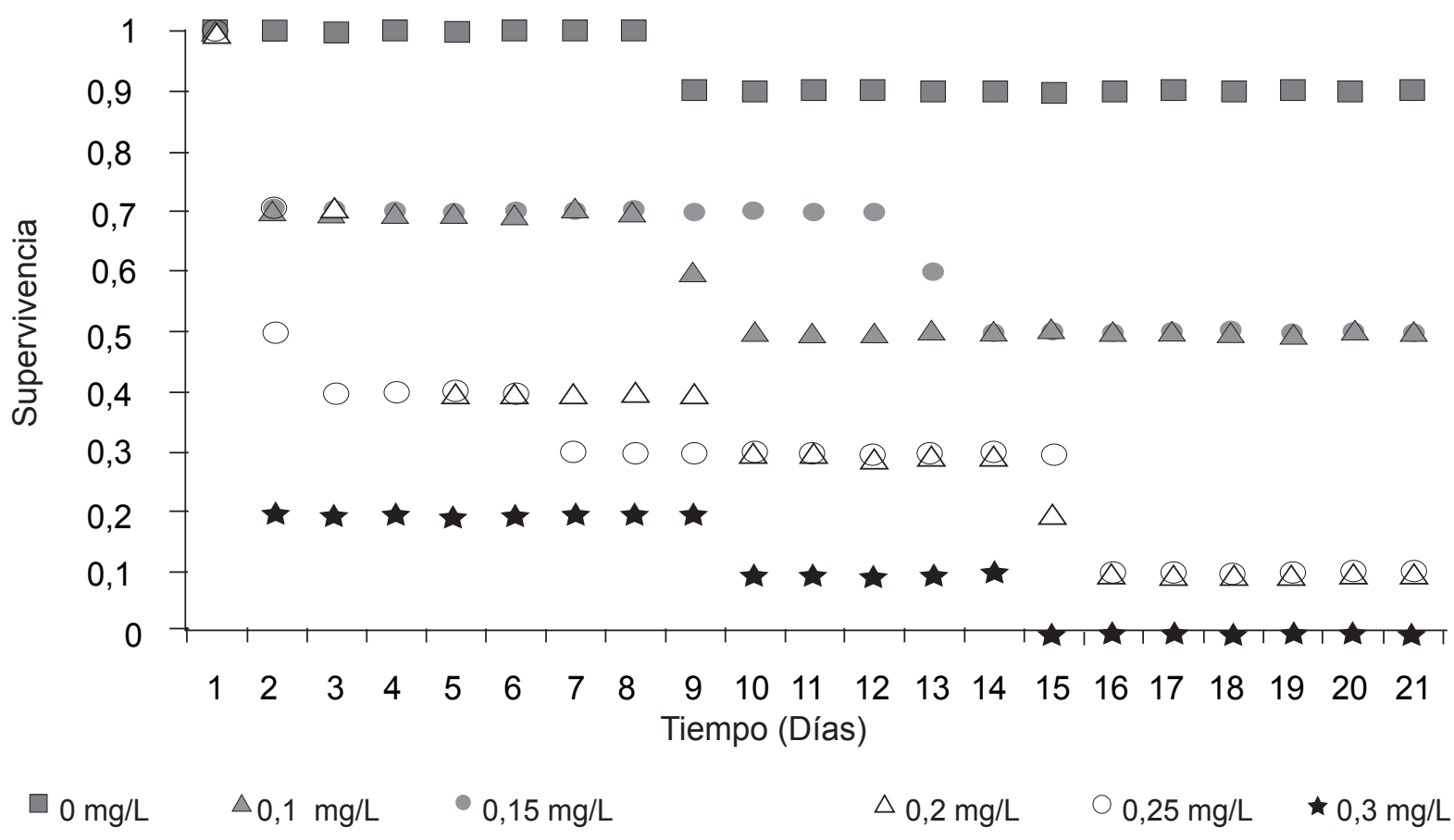

Figura 7. Supervivencia de Ceriodaphnia cornuta expuesta a diferentes concentraciones de lindano. 


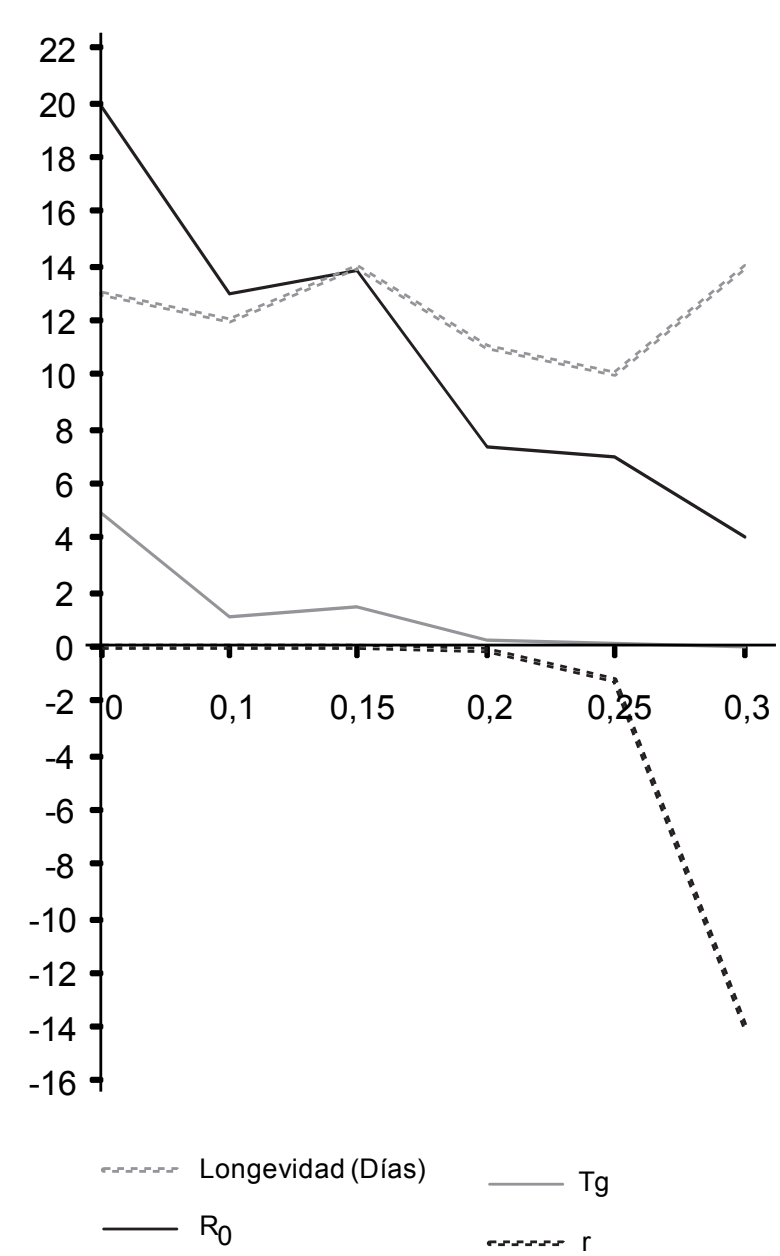

Figura 8. Parámetros demográficos de Ceriodaphnia cornuta expuesta a diferentes concentraciones de lindano.

de $\mathrm{R}_{0}$ y r como también la correspondencia a sus cambios de la longevidad y el tiempo generacional. Los resultados de toxicidad crónica obtenidos demostraron que a medida que aumenta la concentración de lindano, el tiempo que transcurre desde el inicio de la prueba hasta que se produce la primera reproducción de los organismos, aumenta gradualmente. Durante los 21 días de duración del ensayo, se observó una significativa disminución de neonatos producidos al incrementar la concentración del plaguicida. En tal sentido, entre el control y la primera concentración se registra una disminución con una producción del $46 \%$ en la camada. Villarroel Utrillas (2004) observó resultados similares con los organoclorados Tetradifón y Propanil en $D$. magna, más relevante para el segundo tóxico. La longevidad media también se redujo de 19,8 a 4 días (control - máxima concentración), en forma similar a los efectos del insecticida Diazinon (Wong 1997) sobre Moina macrocopa con valores de 5,8 a 1,6 días.

La tasa intrínseca de crecimiento natural (r) resultó ser un parámetro muy sensible al tóxico, ya que si bien existen antecedentes sobre la disminución de su valor entre el control y la máxima concentración, tales como, de 0,22 a 0,17 para $D$. longispina y D. magna expuestas por 21 días a $607,5 \mu \mathrm{g} / \mathrm{L}$ de lindano (Antunes et al. 2004); 0,33 a 0,14 en D. magna después de la exposición a $0,55 \mathrm{mg} / \mathrm{L}$ del herbicida Propanil (Villarroel Utrillas et al. 2003); 0,25 a -0,7 para $D$. pulex expuesta a concentración de $2 \mu \mathrm{g} / \mathrm{L}$ del organofosforado Diazinon (Stark et al. 2003); los valores obtenidos por nosotros son altamente relevantes ya que a máxima concentración, r registró -13,97, evidenciándose la alta mortalidad respecto a la natalidad, en contraposición del control $(0,02)$. Así mismo, podemos inferir que el lindano tiene un efecto negativo sobre la supervivencia de C. cornuta (Fig. 7).

Los resultados obtenidos corroborarían la mayor eficiencia de las especies de Ceriodaphnia en bioensayos. Ceriodaphnia cornuta resultó ser más sensible al lindano con un valor de $\mathrm{CL}_{50} 48 \mathrm{~h}$ $(5,260 \pm 0,7 \mu \mathrm{g} / \mathrm{mL})$ menor que los encontrados en Simocephalus

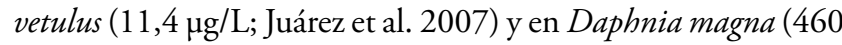
$\mu \mathrm{g} / \mathrm{L}$; Silva et al. 2001 y Martins et al. 2007), indicándolo como un adecuado organismo biosensor de contaminantes.

El tiempo que transcurre desde el inicio de la prueba hasta que se reproduce por primera vez $C$. cornuta aumenta gradualmente a medida que aumenta la concentración de lindano. También se observó una significativa disminución de neonatos al incrementar la concentración del plaguicida. Villarroel Utrillas (2004), observó resultados similares producidos por tetradifón y propanil en Daphnia magna, ambos plaguicidas causaron una disminución progresiva en el número de descendientes, que fue más relevante con el propanil.

El efecto observado sobre la longevidad es relevante, parámetro que decrece considerablemente a mayor concentración de lindano respecto del control. El promedio de longevidad también se reduce de 12,5 días bajo condiciones control a 7,6 días en la máxima concentración del insecticida endosulfan sobre el cladócero Moina macrocopa (Chuah et al. 2007) y de 5,8 a 1,6 días para el Diazinon (Wong 1997).

En cuanto a la tasa intrínseca de crecimiento natural (r), los valores obtenidos demuestran también un importante efecto del lindano a máxima concentración $(-13,97)$ respecto del control $(0,02)$. Antunes et al. (2004) registraron para este parámetro valores de 0,22 y 0,17 para $D$. longispina y $D$. magna expuestos por 21 días a $607,5 \mu \mathrm{g} / \mathrm{L}$ de lindano, respectivamente. En ensayos con los insecticidas organofosforados spinosad y diazinon sobre $D$. pulex se observó que la tasa de incremento natural declinó dependiendo de la concentración (Stark et al. 2003). Así mismo (r) resultó ser el parámetro más sensible afectado por la toxicidad del herbicida propanil en $D$. magna desde 0,33 para el control a 0,14 después de la exposición a $0,55 \mathrm{mg} / \mathrm{L}$. (Villarroel Utrillas et al. 2003). Los resultados obtenidos de la toxicidad del plaguicida organoclorado lindano sobre los parámetros reproductivos de C. cornuta son importantes indicadores del impacto de estos sobre la biota acuática.

\section{Agradecimientos}

Este trabajo se realizó gracias al subsidio recibido del Proyecto CIUNT (Consejo de Investigaciones de la Universidad Nacional de Tucumán). 26/G446-1- "Ecología de Humedales del Noroeste Argentino con especial énfasis al Impacto Ambiental" (2008 - 2012).

\section{Literatura citada}

Antunes S., B. Castro \& F. Gonçalves. 2004. Effect of food level on the acute and chronic responses of daphnids to lindane. Environmental Pollution 127: 367-375.

Avalos Gómez M. \& J. Ramírez Gutiérrez. 2003. La situación del lindano en México. Gaceta Ecológica. Instituto Nacional de Ecología 69: 93-100. 
Chuah T. S., J. Y. Loh \& Y. S. Hii.2007. Acute and Chronic Effects of the Insecticide - Endosulfan on Freshwater Cladoceran, Moina macrocopa Straus. Bull. Envirom. Contam. Toxicol. 79: 557- 561.

Giorgio A. \& A. Digón. 2005. Sustancias o Compuestos Tóxicos Químicos Prohibidos o Restringidos en la República Argentina. Programa Nacional de Riesgos químicos. Pp. 15.

Iannacone J., L. Alvariño, C. Caballero \& J. Sánchez. 2000. Cuatro Ensayos Ecotoxicológicos para Evaluar Lindano y Clorpirifos. Gayana 64(2): 139-146.

INE (Instituto Nacional de Ecología). 2004. El Lindano en México. Instituto Nacional de Ecología Pp. 56.

ISO (International Standards Organization) 1982. Water QualityDetermination of the inhibition of the Mobility of Daphnia magna Straus (Cladocera, Crustacea). 1st Ed. E. Geneve, Switzerland 1982-03-15. Ref. ISO 6341.

Juárez J. \& A. Villagra de Gamundi. 2007. Bioensayos preliminares para evaluar la toxicidad del lindano sobre Simocephalus vetulus (O.F.Muller, 1776) (Crustacea: Cladocera). Revista Peruana de Biología. 14(1): 65 - 67. Versión Online ISSN: 1727 - 9933.

Martins J., L. Oliva Teles \& V. Vasconcelos. 2007. Assays with Daphnia magna and Danio rerio as alert systems in aquatic toxicology. Environmental International. 33: $414-425$.
Silva J, J. Iannacone, A. Cifuentes, et al. 2001. Assessment of Sensitivity to Pentachlorophenol (PCP) in 18 Aquatic Species, Using Acute and Chronic Ecotoxicity Bioassays. Ecotoxicology and Environmental Restoration 4(1): 10 - 17.

Stark J. \& R. Vargas. 2003. Demographic changes in Daphnia pulex (Leydig) after exposure to the insecticides spinosad and diazinon. Ecotoxicology and Environmental Safety 56: $334-338$.

USEPA 1988. Short_Term methods for estimating the chronic toxicity of effluents and receiving waters to marine and estuarine organisms. EPA/600/4-87/028. Pp. 398 - 416.

Villarroel Utrillas M. J., E. Sancho, M. D. Ferrando E. Andreu. 2003. Acute, chronic and sublethal effects of the herbicide propanil on Daphnia magna. Chemosphere 53: 857 - 864.

Villarroel Utrillas M. J. 2004. Tesis Doctoral: Alteraciones Fisiológicas en el Crustáceo Daphnia magna por exposición a plaguicidas. Server de Publicacions. Universitat de Valencia. Pp. 212.

Villalobos M. 2006. Estudios sobre la Biología y Ecología de Ceriodaphnia cornuta Sars. Una revisión. Interciencia. 31 (005): $351-357$.

Wong C. K. 1997. Effects of Diazinon on some population parameters of Moina macrocopa (Cladocera). Water, Air, and Soil Pollution 393: 393 - 399. 